\title{
Developing a macroscopic model based on fuzzy cognitive map for road traffic flow simulation
}

\author{
Mehran Amini $^{1 *}$, Miklos F. Hatwagner ${ }^{2}$, Gergely Mikulai ${ }^{3}$, and Laszlo T. Koczy
}

\begin{abstract}
Fuzzy cognitive maps (FCM) have been broadly employed to analyze complex and decidedly uncertain systems in modeling, forecasting, decision making,etc. Road traffic flow is also notoriously known as a highly uncertain nonlinear and complex system. Even though applications of FCM in risk analysis have been presented in various engineering fields, this research aims at modeling road traffic flow based on macroscopic characteristics through FCM. Therefore, a simulation of variables involved with road traffic flow carried out through FCM reasoning on historical data collected from the e-toll dataset of Hungarian networks of freeways. The proposed FCM model is developed based on 58 selected freeway segments as the "concepts" of the FCM; moreover, a new inference rule for employing in FCM reasoning process along with its algorithms have been presented. The results illustrate $\mathrm{FCM}$ representation and computation of the real segments with their main road traffic-related characteristics that have reached an equilibrium point. Furthermore, a simulation of the road traffic flow by performing the analysis of customized scenarios is presented, through which macroscopic modeling objectives such as predicting future road traffic flow state, route guidance in various scenarios, freeway geometric characteristics indication, and effectual mobility can be evaluated.
\end{abstract}

Index Terms-Fuzzy cognitive map, road traffic flow, macroscopic model

\section{INTRODUCTION}

$\mathrm{T}$ raffic related issues have significant environmental, economic, and social consequences, including air pollution, the reduction of effectual mobility, the increase of fuel and time waste, etc. These problems can be mitigated to maintain citizens' safety, to balance the demand-capacity congestion ratio, and to reduce the cost related congestion through a wide range of methods from detecting frequent traffic congestions by using spatial congestion propagation patterns [1], to create intelligent traffic lights controller algorithms and cooperative scheduling [2]. Analyzing and modeling road traffic flowassociated parameters are the main aims of these methods. Modeling these parameters (e.g., density, time, velocity) is seen as indispensable to comprehend the heterogeneous behavior of road traffic [3], [4], even though it is difficult due to the nonlinearity and uncertainty caused by internal and external elements, for example, drivers' preferences, weather conditions, imprecision in the collected data by sensors [5].

\footnotetext{
${ }^{1,2}$ Department of Information Technology, Szechenyi Istvan University, Gyor, Hungary

${ }^{3}$ Doctoral School of Regional Sciences and Business Administration, Gyor, Hungary

${ }^{4}$ Department of Information Technology, Szechenyi Istvan University, Gyor, Hungary and Department of Telecommunications and Media Informatics, Budapest University of Technology and Economics, Hungary
}

Modeling these nonlinear and uncertain characteristics becomes more applicable by the development of intelligent transportation systems (ITS) and soft computing (SC) techniques [6].

The field of intelligent transportation systems arose in the early 1950 s through the combination of multidisciplinary techniques such as information technology, electronics, and traffic engineering, in order to deal with transportation-related problems more efficiently by new data inference and communication tools [7]. Such systems mainly aim at enhancing the productivity of the current transportation systems in order to avoid traffic breakdowns and the traffic shifting from uncongested to a congested state. All these initiatives have similar characteristics; namely, first, they all seek to understand the essence of the road traffic flow at a particular location and then control its alterations. Thus, both rely mainly on conventional statistics-based approaches, e.g., Bayesian network models, nonparametric regression, history average, and autoregressive integrated moving average. These techniques are often unable to completely address the complexities associated with involved parameters of traffic and their relationships and mainly resulting in unreliable road traffic detection and prediction [8], [9].

By introducing self-learning data processing techniques rather than model-based estimation methods caused by the advancement in inferential intelligence, data-driven approaches have developed rapidly [10], [11]. The emphasis of the classical numeric methods is on assuming certain statistical behaviors of the system in advance, mainly based on stationary and deterministic features. Hence, they fail to model the complex, non-deterministic, uncertain behavior of the system, where intelligent self-learning data processing techniques could be able to model the complexity of the system on hand, based on understanding the available data to build up an adequate structure. This understanding of the system is achievable by sacrificing completeness and accuracy and by tolerating imprecision in order to attain tractability, cognition, and costeffective solutions [12], [13]. Zadeh named the various methodologies based on intelligence and sub-symbolic representation of the phenomena 'Soft Computing' (SC) [14]. Recently, soft computing techniques such as fuzzy-based inference, neural networks, evolutionary and population-based computing, such as swarm intelligence, etc., have provided significant achievements in improving the performance of ITS. These enhancements are achieved mainly due to the massive changes in the data scale generated and collected from various sources by the involved stakeholders, e.g., governments, 
citizens, and the industry with respect to these systems [15], [16]. Intelligent transportation-based systems are indeed a wellsuited area to apply soft computing techniques since the data provided here are full of uncertainty and vagueness, where technical disciplines of SC techniques such as approximate computing and randomized search can be properly employed [17], [18]. In previous studies, SC methods have been proposed in various transportation problems such as road traffic flow and state prediction in [8] and [17], vehicle route planning, and vehicular ad-hoc networks in [20]. Thus far, the abilities of SCbased techniques in terms of modeling the road traffic flow in networks of freeways have been more or less neglected.

Moreover, in traffic control engineering projects, the road traffic flow modeling has significant contributions, take for instance, strategy assessment and development for road traffic control management, the inspection, and forecast of road traffic conditions in dynamic networks in the short term, evaluating the effect of recent constructions and comparing alternatives, etc. [21], [22]. With regard to road traffic flow characterization, three classes representing three levels of the models have been applied: the macroscopic, microscopic, and mesoscopic levels [23]. At the macroscopic level, aggregate road traffic is modeled by global variables, i.e., velocity, density, and flow of the road traffic as a mass behavior, while individual vehicle behavior is considered at the microscopic level only [24]. Both aggregate and individual behaviors are analyzed at the intermediate mesoscopic level [25]. The first macroscopic road traffic flow model was introduced by Lighthill [26]; since then, these models have gained increasing attention because of their uncomplicatedness and low inferential complexity, the latter enabling real-time evaluation and actions. This study aims at introducing a new macroscopic model based on fuzzy cognitive maps as one of the SC techniques for networks of freeways simulation.

Kosko defined Fuzzy Cognitive Maps (FCM) as: "fuzzy feedback models of causality that combine aspects of fuzzy logic, neural networks, semantic networks, expert systems, and nonlinear dynamical systems" [27]. Since then, a wide range of FCM applications have been conducted, see i.e., [28], [29]. One of the most frequent applications of FCM is in describing and simulating systems, including uncertainty and imprecision [17], [30]. Although FCM applications in the risk analysis area based on the concepts of failure, incident, error, etc. have been proposed already [31], the research effort described here has primarily been to model uncertain and non-deterministic conditions of heterogeneous road traffic flow systems through developing a macroscopic level-based method. The proposed FCM model also leads to illustrating the key arguments supporting the approach based on FCM, i.e., the sophistication and the efficient computational effort. Accordingly, this paper is devoted to demonstrating the abilities of FCM in modeling road traffic flow based on historical data collected from the networks of freeways in Hungary. This approach will lead to predicting the future states of road traffic flow, some indications concerning the geometric and geographic characteristics of the freeways, and the overall behavior of the network in various road traffic scenarios.
The rest of the paper is outlined as follows. In the next section, various road traffic flow models along with an introduction of METANET and FCM as the basis of the proposed model are highlighted. The third section presents the proposed new method with implementation aspects. Following that, the description of the applied dataset is given and the steps of the proposed new algorithm are defined and elaborated. In the fourth section, the performance of the proposed model's results is investigated. Some conclusions are presented in the fifth and last sections.

\section{MODELS}

The necessity of modeling road traffic flow was raised because of the importance of mathematically describing the dynamic and complex behavior of road traffic-related systems. The first theoretic model of road traffic flow was introduced in [32]. Since then, a variety of road traffic flow-based models with different properties have been proposed. These models have been developed for various aims ranging from system analysis and future state forecasting to the modification of the current infrastructures. Categorizing these models is mainly based on two factors, the level of details coupled with the differentiation between macroscopic, microscopic, and mesoscopic methods [33]. In this study, the model's focus is narrowed down on discrete macroscopic characteristics, which lays emphasis on the overall behavior of vehicles over time. As well as the involved variables are discretized (both temporally and spatially) instead of using continuous variable, i.e., freeways are considered as a set of segments with defined lengths, and time is also divided into discrete intervals [34].

Subsequently, a generic integrated approach in Section Three is presented; as a matter of fact, the approach not only can be applied to modeling macroscopic road traffic flow, but it also illustrates the potential application of fuzzy cognitive maps in modeling complex and nonlinear systems, which are known notoriously as full of uncertainty and imprecision. This unified approach is presented by employing two particular models: METANET [35] from the class of macroscopic road traffic flow models and the fuzzy cognitive map approach [36] as a soft computing method through which recognizing, classifying, and modeling complex systems is a possible approach.

\section{A. METANET}

METANET was introduced as a program to simulate freeway networks in a macroscopic way [35]. This simulation of the road traffic behavior in networks of freeways is based on an overall road traffic flow modeling that was originally developed by Payne [37]. METANET, as the most recognized secondorder macroscopic approach, has been used in engineering and control-related problems. Second-order approaches lay emphasis on vehicles density and velocity by characterizing them in dynamic equations [5]. These properties allow a reasonably low-time inferential process. Therefore, real-time network simulation and representation are efficiently possible. The freeway network is embodied by a directed graph, i.e., bifurcations and junctions are represented by the nodes of the graph, while the freeway sections between these places are 
Developing a macroscopic model based on fuzzy

cognitive map for road traffic flow simulation characterized by the edges (links). A freeway with two directions is modeled as two distinct directed edges with reverse directions. Edges are assumed to possess homogeneous geometric properties, e.g., the number of lanes is fixed. On the other hand, heterogeneous freeways may be modeled by connected edges separated by nodes at the places where the change of geometry happens [35]. Nonlinear difference equations are reflected in the model to illustrate the evolution of the road traffic associated variables, i.e., average space-mean velocity $v(\mathrm{~km} / \mathrm{h})$, average density $\rho$ ( $\mathrm{veh} / \mathrm{km} / \mathrm{lane})$, and average flow $q(\mathrm{veh} / \mathrm{h})$.

In the METANET simulation, whenever the geometry of freeway changes, e.g., a lane rises or drops, a junction, etc., a node is added to the model. Connections among these nodes are called links. Afterward, links are separated into equal segments. The following are the essential equations that are employed for determining the road traffic variables for each segment $i$ of link $m[5]$.

$$
\begin{aligned}
& q_{m, i}(k)=\lambda_{m} \rho_{m, i}(k) v_{m, i}(k) \\
& \rho_{m, i}(k+1)=\rho_{m, i}(k)+\frac{T_{s}}{L_{m} \lambda_{m}}\left[q_{m, i-1}(k)-q_{m, i}(k)\right] \\
& v_{m, i}(k+1)=v_{m, i}(k)+\frac{T_{s}}{\tau}\left[V\left[\rho_{m, i}(k)\right]-v_{m, i}(k)\right. \\
& +\frac{T_{s} v_{m, i}(k)\left[v_{m, i-1}(k)-v_{m, i}(k)\right]}{L_{m}}-\frac{T_{s} \eta\left[\rho_{m, i+1}(k)-\rho_{m, i}(k)\right]}{\tau L_{m}\left(\rho_{m, i}(k)+\kappa\right)} \\
& V\left[\rho_{\mathfrak{m}, i}(k)\right]=v_{\text {free }, m} \exp \left[-\frac{1}{b_{\mathfrak{m}}}\left(\frac{\rho_{m, i}(k)}{\rho_{c r, m}}\right)^{\left.b_{\mathfrak{m}}\right]}\right.
\end{aligned}
$$

where $q_{\mathrm{m}, i}(k)$ represents the outflow of segment $i$ in link $m$ over the time frame $\left[k T_{s},(k+1) T_{s}\right], v_{m, i}(k)$ and $\rho_{\mathfrak{m}, i}(k)$, signify space-mean speed (average speed of vehicles passing a segment during a time period) and the density of segment $i$ of link $m$ at time frame $k$, respectively. $L_{m}$ represent the lengths of the segments situated in link $m$, while $\lambda_{m}$ is the number of lanes in link $m$, and $T_{s}$ represents the simulation discrete time frame. In Eq. (3), $\tau, \eta$, and $\kappa$ are global variables with constant values for all links in the freeway. They are named time constant, anticipation constant, and model parameter, respectively. Additionally, $\rho_{c r, m}$ as critical density, $b_{m}$ as the parameter of the fundamental diagram, and $v_{\text {free, } m}$ as free-flow speed are specific for the basic diagram of the computed link $m$ [5], [35].

\section{B. The Fuzzy Cognitive Map approach}

As an advancement of classic cognitive maps [38], the concept of the fuzzy cognitive map was introduced by Kosko [36] for the purpose of dealing with shortcomings related to the binary nature of the original cognitive map model. FCMs integrate the model of cognitive maps, and the idea of fuzzy set proposed originally by Zadeh [39]. They contain fuzzy nodes or concepts to explain the non-binary states of the modeled system components (concepts) as well as the gradual intensities of causalities among them. Although both models are represented as directed and signed graphs, the causal mechanisms with imprecise causal data can be described adequately only by the FCM. In this approach, more human-like reasoning in complex dynamic systems is applied, both in the model structure and the related computational processes. A schematic illustration of the FCM is indicated in Fig. 1; connections and interrelationships among concepts are modeled with weighted arcs.

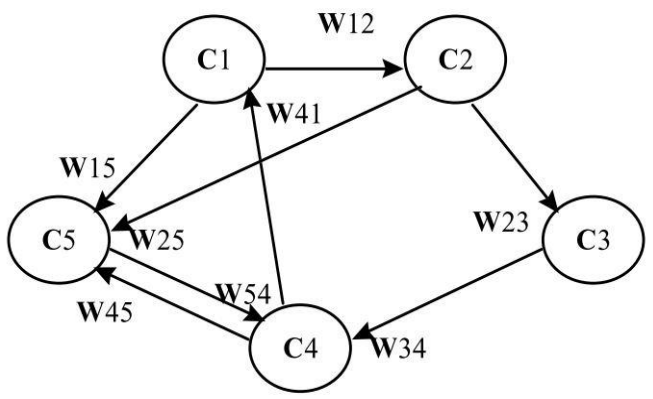

Fig. 1 A schematic illustration of simple FCM [7]

As it can be seen (Fig. 1), the variables of the system are represented by the indicated nodes $C_{I}$ to $C_{5}$. These variables are known as cause concepts where include nodes at the origin points of the arcs as well as effect concepts, where located at the terminal points of arcs. Take for instance, the $C_{1} \rightarrow C_{2}$ connection, where $C_{l}$ is the cause variable because of impacting on $C_{2}$ as the effect variable. All concepts are individually identified by a number $A_{i}$ commonly in the interval $[0,1]$, which signifies its value in the model. Considering the signed (bipolar) fuzzy interval $[-1,1]$ enables the model to assign grades or degrees of causality to the connections among the concepts [40]. The type of connection between two concepts signifies the influence of one concept $\left(C_{i}\right)$ upon another one $\left(C_{j}\right)$, where the interaction between them can be interpreted as excitatory or positive causality $(w i j>0)$, and inhibitory or negative causality $(w i j<0)$; and finally, null or no connection $(w i j=$ 0 ). Hence, the behavior of the system is warehoused and reflected in the structure of the concepts and the respective interconnections among them[41], [42].

Eq. (5) indicates the first introduced inference rule for the fuzzy cognitive map with $A^{(0)}$ as the initial activation vector; then the new activation vectors are computed at every individual step $t$ and after defining the number of iterations after which the model will reach either its equilibrium point, or, the so-called limit cycle, or, eventually a chaotic behavior. The model shows these states under the following circumstances [36], [43], [44]:

- it stabilizes at fixed numerical values, achieving equilibrium at a fixed-point attractor with output values that are decimals in the interval.

- it displays limit cycle behavior, with output values falling into a loop of numerical values over a set time period.

- it illustrates a chaotic behavior, with each output value reaching a wide range of numerical values in a random, non-periodical, and non-deterministic manner.

Thus, updates are iteratively introduced until a terminal state has been reached. In this procedure, a state vector containing the activation degrees of the involved concepts is produced by the FCM at everv discrete time frame $\lceil 291$. 


$$
A_{i}^{(t+1)}=f\left(\sum_{\substack{j=1 \\ i \neq j}}^{n} w_{j i} A_{j}^{(t)}\right)
$$

Although Eq. (5) had been employed in many FCM applications as the inference rule, a revised updating rule was introduced in [45], which is presented in Eq. (6), where the concept also considers its past value. The concepts are taken into account first, by their previous activation values, and second, by the activation values provided by other concepts and their corresponding weights.

$$
A_{i}^{(t+1)}=f\left(\sum_{\substack{j=1 \\ i \neq j}}^{n} w_{j i} A_{j}^{(t)}+A_{i}^{(t)}\right)
$$

Various computation rules have been proposed [46], [47]. Applying the appropriate computational rule is determined by the type of the problem. Thus, the problem needs a profound understanding of all involved aspects before these rules are set. In both Eqs. (5) and (6), $f$ is a threshold (squeezing) function. It expresses a monotonically non-decreasing function that defines the activation value of every concept toward the desired interval $I$, where either $I=[0,1]$ or $I=[-1,1]$, determined by the actual domain. The most broadly employed transfer functions are the bivalent, the saturation, or the trivalent, hyperbolic tangent, and sigmoid functions.

$$
f(x)=\frac{1}{1+e^{-\lambda(x-h)}}
$$

The sigmoid function is given in Eq. (7). It is a continuous transfer function that provides an illimitable number of various states that are distributed within the desired hypercube. In the sigmoid transfer function, $\lambda>0$ and $\mathrm{h}>0$ are user-defined parameters adjusting the function slope and offset, respectively. Greater values of $\lambda$ raise the steepness, and it controls responsiveness to the variations of $x$. Fig. 2 shows the effect of the choice of value of $\lambda$ in the transformation or inference results. Besides, increasing the activation value leads to the growth of the derivative [29].

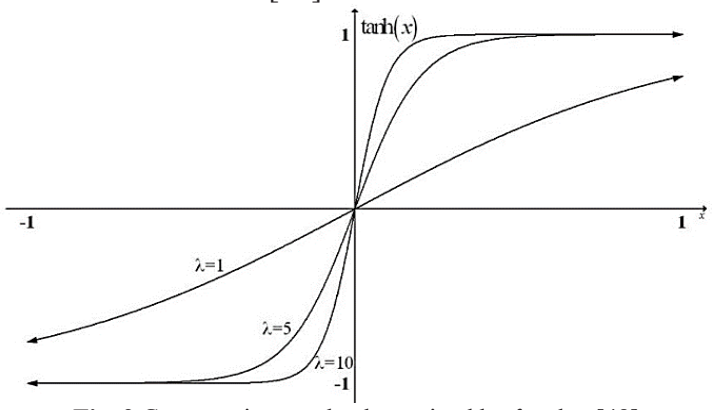

Fig. 2 Computation results determined by $\lambda$ value [48]

\section{THE PROPOSED NEW METHOD}

In the sequel, an approach is presented which can integrate the macroscopic method with the fuzzy cognitive map approach to model road traffic flow. FCMs can be seen as recurrent neural networks with inference features, which include a set of neural computing entities or concepts [41]. Defining activation values for these concepts coupled with weight assignments is an essential part of creating the road traffic flow model based on FCM. In the proposed model, the activation values are assigned by an inference rule that is determined by combining the highlighted equations in Table 1. Therefore, it can be observed that the emphasis of the proposed integration is on two important factors; not only can activation values be computed by the values of the linked concepts with the corresponding causal weights at each time step, but concepts also take into account their own previous values. Algorithm steps will be elaborately explained in the implementation steps after

\begin{tabular}{|c|c|c|}
\hline Author/s & Equation/Method & Usage \\
\hline [35] & $\begin{array}{l}\rho_{m, i}(t+1) \\
=\rho_{m, i}(t)+\frac{T_{s}}{L_{m} \lambda_{m}}\left[q_{m, i-1}(t)\right. \\
\left.-q_{m, i}(t)\right]\end{array}$ & $\begin{array}{l}\text { Computing the } \\
\text { future density of } \\
\text { segment } i \text { (i.e., } \\
\text { various sections with } \\
\text { specific length } \\
\text { between 100-18000 } \\
\text { m) of link } m \text { (i.e., } \\
\text { homogeneous } \\
\text { freeway consist of } \\
\text { several segments) at } \\
\text { simulation time step. }\end{array}$ \\
\hline [45] & $A_{i}^{(t+1)}=f\left(\sum_{\substack{j=1 \\
i \neq j}}^{n} w_{j i} A_{j}^{(t)}+A_{i}^{(t)}\right)$ & $\begin{array}{l}\text { Computing the value } \\
\text { of concept } C_{i} \text { at time } \\
t \text {, that the value of } C_{i} \\
\text { is the calculated } \\
\text { density in the } \\
\text { segment (section) of } \\
\text { the given link } \\
\text { (freeway). }\end{array}$ \\
\hline
\end{tabular}
describing the dataset.

TABLE I: INVOLVED INDICES/METHODS IN THE PROPOSED INFERENCE RULE

\section{A. Data description}

The presented FCM model was trained on road traffic data of the Hungarian network of freeways. Freeway users in Hungary experience complex and dynamic patterns of congestion. Besides other reasons, e.g., rather intensive road traffic caused by Hungary's strategic location in the European transport network and the system of corridors [49], this is mainly because of the increasing number of registered vehicles is Hungary, i.e., an increase of around 25\% from 2010 to 2018 [50]. These problems lead to complex behavior with temporal and spatial alterations in road traffic. Therefore, modeling vehicles flow by available resources is seen as indispensable.

The dataset is collected from the online transaction processing server of the Hungarian e-toll system, which is an electronic system operated by the Hungarian national toll payment services for the whole network of motorways and primary highways of the country. This system enables the assistance and support of the verification of freeways usage, admittance, levying, and finally collecting the tolls of the standard road sections tollways [51]. The dataset contains seven variables: the name of the freeway, the section name (identifier), the collected e-toll over a span of one week in each section (segment) of the 212 freeway sections (links), which latter is considered as a proportional indicator of the number of vehicles, the time (per minute), the day, the length of the sections, and the number of the lanes in each section. These links include 2446 different segments altogether. Each segment length varies from 100 to 18,000 meters. For the sake of reducing the complexity of the 
model, a sample of 58 segments was selected, the full set of freeway sections through which Budapest is connected to the Austrian border.

Most of the road traffic models' aim is to explain the behavior of traffic-involved variables over the full range of operation, where identified locations have a pivotal role in the investigated dataset. This dataset can represent road traffic behavior based on location in a real-time manner. In Fig. 3, a sample of connections among three segments $\mathrm{A}, \mathrm{B}$, and $\mathrm{C}$, are illustrated. Since the available dataset is based on time series, therefore, current traffic state in upstream segments can characterize road traffic flow conditions in downstream segments in the next time frames.

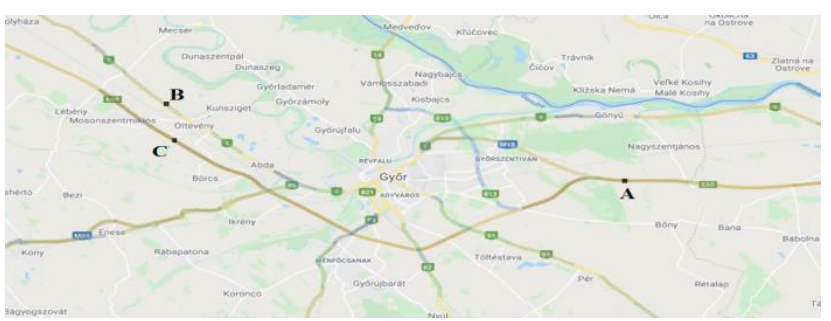

Fig. 3 A sample of freeway network and segment connections

The above-mentioned segments' causal relationships and correlations can be observed in Fig. 4. In the horizontal axis, the first digit represents the day, while the second and third digits represent the hour (in 24-hour format); an accurate behavior of road traffic flow over time is indicated, showing how traffic flow in the upstream segment can affect the subsequent segments. The calculated road traffic flow correlation among segments reveals that the correlation of $\mathrm{A}$ and $\mathrm{B}$ is 0.03 , between $\mathrm{A}$ and $\mathrm{C}$ it is 0.9 , and $\mathrm{B}$ and $\mathrm{C}$ correlate to 0.1 ; through which values various conclusions and correlation analyses can be conducted to identify the behavior and intensity of road traffic flow.

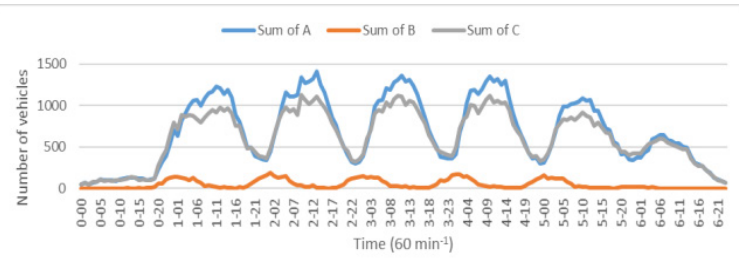

Fig. 4 Causal relationships of road traffic streamflow of three sample segments

\section{B. The Model}

The key deficit of applying FCM is the critical reliance on the initial expert judgment [52]. This issue stands out, particularly in modeling complex systems. In this research, extracted parameters of a macroscopic road traffic model have been applied for assigning initial values of concepts and weights. Each road segment is represented by a concept whose value is considered as the density $\rho$ of segment $i$ of link $m$, and the weighted arcs are set to a constant value based on $L_{m} \lambda_{m}$ variables as approximate capacity; where $L_{m}$ denotes the length of the segments of link $m, \lambda_{m}$ denotes the number of lanes of link $m$. The concepts and the weights initializations are set based on the aforementioned values. Afterward, the system is allowed to interact, and after every iteration, the new state vector is assigned newly generated values. This procedure will continue until the model exhibits an equilibrium state by reaching a stabilized condition at a fixed numerical boundary. The macroscopic road traffic model based on FCM is presented by the proposed algorithm in Fig. 5.

\begin{tabular}{|c|c|}
\hline \multicolumn{2}{|c|}{ Simulation steps } \\
\hline \multicolumn{2}{|c|}{ • Preprocessing: } \\
\hline Step 1 & $\begin{array}{l}\text { Importing the dataset, consisting of seven columns: } m \text {, is the link } \\
\text { name, } i \text {, is the segment name, } q_{\mathfrak{m}, i} \text {, is the no. of vehicles, } T_{s} \text {, the } \\
\text { time day, } L_{m} \text { the segment length, and } \lambda_{m} \text {.the no. of segment } \\
\text { lanes }\end{array}$ \\
\hline Step 2 & $\begin{array}{l}\text { Calculating and adding the density (concept values) as the eighth } \\
\text { column according to the equation: } \\
\qquad \rho_{m, i}=\frac{n_{t}}{L_{m} \lambda_{m}}\end{array}$ \\
\hline Step 3 & $\begin{array}{l}\text { Calculating } L_{m} \lambda_{m} \text { as weight values initialization and adding the } \\
\text { results as the ninth column to the dataset. }\end{array}$ \\
\hline Step 4 & $\begin{array}{l}\text { Normalizing and adjusting concepts (density) and weights } \\
\left(L_{m} \lambda_{m}\right) \text { in the }[0,1] \text { interval scale: } \\
\qquad X_{\text {normalzed }}=\frac{\left(X-X_{\min }\right)}{\left(X_{\max }-X_{\min }\right)}\end{array}$ \\
\hline Step 5 & $\begin{array}{l}\text { Defining connection weight matrix } \mathrm{W}_{\mathrm{ij}} \text { as } L_{m i} \lambda_{m i} \text { based on the } \\
\text { sequence of the segments, based on two possible types of causal } \\
\text { relationships among concepts, i.e., when road traffic streams } \\
\text { from concept } C_{i} \text { to } C_{j} \text { : } \\
\qquad \quad \mathrm{W}_{\mathrm{ij}}>0 \text { excitatory causality } \\
\text { - } \quad \mathrm{W}_{\mathrm{ij}}<0 \text { inhibitory causality }\end{array}$ \\
\hline Step 6 & $\begin{array}{l}\text { Transforming equation } 1 \text { to } 2 \text { for calculating the value of concept } \\
\mathrm{C}_{\mathrm{i}} \text { as the density of segment } i \text { at time t: }\left(\rho_{m, i}^{t}\right) \text {. } \\
\text { 1) } A_{i}^{(t+1)}=f\left(\sum_{\substack{j=1 \\
i \neq j}}^{n} W_{i j} A_{j}^{t}+A_{i}^{t}\right) \\
\text { 2) } \rho_{m, i}^{t+1}=f\left(\sum_{\substack{j=1 \\
i \neq j}}^{n} \rho_{m, i, j}^{t} W_{i j}+\rho_{m, i, i}^{t}\right)\end{array}$ \\
\hline \multicolumn{2}{|c|}{ - Main Algorithm: } \\
\hline Step 7 & Read the input initial concept state (input vector) $\mathrm{A}^{0}$ as $\rho_{m, i}^{0}$. \\
\hline Step 8 & Define the relationship weight matrix $W_{i j}$ \\
\hline Step 9 & $\begin{array}{l}\text { Calculate the concept state } \rho_{m, i}^{t} \text { according to the equation: } \\
\qquad \rho_{m, i}^{t}=f\left(\sum_{\substack{j=1 \\
i \neq j}}^{n} \rho_{m, i, j}^{t-1} W_{i j}+\rho_{m, i, i}^{t-1}\right) \\
\end{array}$ \\
\hline Step 10 & $\begin{array}{l}\text { Apply the threshold function to output vector } \rho_{m, i}^{t}=f\left(\rho_{m, i}^{t}\right) \text { : } \\
\qquad f(x)=\frac{1}{1+e^{-\lambda(x-h)}}\end{array}$ \\
\hline Step 11 & $\begin{array}{l}\text { If }\left(\rho_{m, i}^{t+1}=\rho_{m, i}^{t}\right) \text {, stop; } \\
\text { Else Go To Step 7; } \\
\text { End }\end{array}$ \\
\hline
\end{tabular}

Fig. 5 Simulation steps of macroscopic road traffic flow model based on FCM

\section{RESUlts}

Complex road traffic flow processes are characterized by various dimensions and components that are highly dependent 
and interconnected. For this reason, FCM as a soft computing technique is presented to address networks of freeways included imprecision and uncertainty. These uncertainties from the macroscopic modeling point of view are mainly connected with road traffic flow, density, and approximate capacity associated variables that can increase the probability of a breakdown and shifting the free flow state of traffic to congested flow [11], [34]. According to the applied algorithm in the previous section, segments of each link (freeway) are assigned as the concepts (nodes) of the FCM, where calculated density defines their values. In Fig. 6, a geographical representation of the selected segments is presented. There are 58 segments in the three investigated links, i.e., $S_{1}, S_{2}$, and $S_{3}$. Each of these links can be selected as the possible route from Budapest to the main Hungarian-Austrian border corridor represented as $E . E S_{1}$, and $E S_{2}$ are the endpoints of the $S_{1}$ and $S_{2}$ links, respectively.

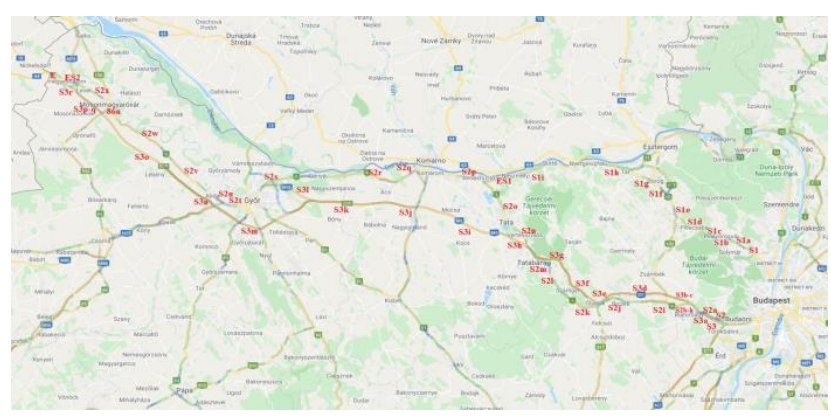

Fig. 6 Geographical locations of the selected segments

Therefore, the concept value initialization as the first step of FCM construction was determined corresponding to the real measured density, i.e., $\rho_{m, i}^{t}$ for segment $(i)$, link $(m)$, and time step $(t)$. This value stands for downstream in the selected segment (transformed in the interval $[0,1]$ ), where the flow has not arrived at the next segment yet. Afterward, the FCM road traffic flow model developed by assigning weight values in accordance with the approximate capacity of each link through $L_{m} \lambda_{m}$. Fig. 7 depicts the initial state of the concepts and their respective interconnections along with the quantified weights in the interval $[-1,1]$, which enables the classification of the degrees of causality among the segments. Two types of interactions among the segments are considered; where one segment $\left(C_{i}\right)$ has excitatory causality on the subsequent segment $\left(C_{j}\right)$, then wij $>0$, which means downstream of $C_{i}$ becomes upstream of $C_{j}$, while $C_{j}$ has negative causality on $C_{i}$ signified by a causal edge with a negative value from $C_{j}$ to $C_{i}$. Consequently, the behavior of the segments coupled with interconnections among them is reflected and warehoused in the FCM thus constructed.

In Fig. 7, three alternative links that can be chosen from Budapest to the Austrian border are illustrated by $S_{1}, S_{2}$, and $S_{3}$ and their 58 nodes in the network. $S_{1}$ includes nine segments that end at segment $E S_{1}$ and joins to one of the $S_{2}$ segments; $S_{3}$, as the most chosen route, also has close interaction with the segments in $S_{2}$, which both end at segment $E$ as the last Hungarian segment before entering Austrian territory. Greater activation values in the concepts (segments) are indicated by larger nodes in the modeled FCM; they represent greater density and show stronger activation values that cause greater impact on the network.

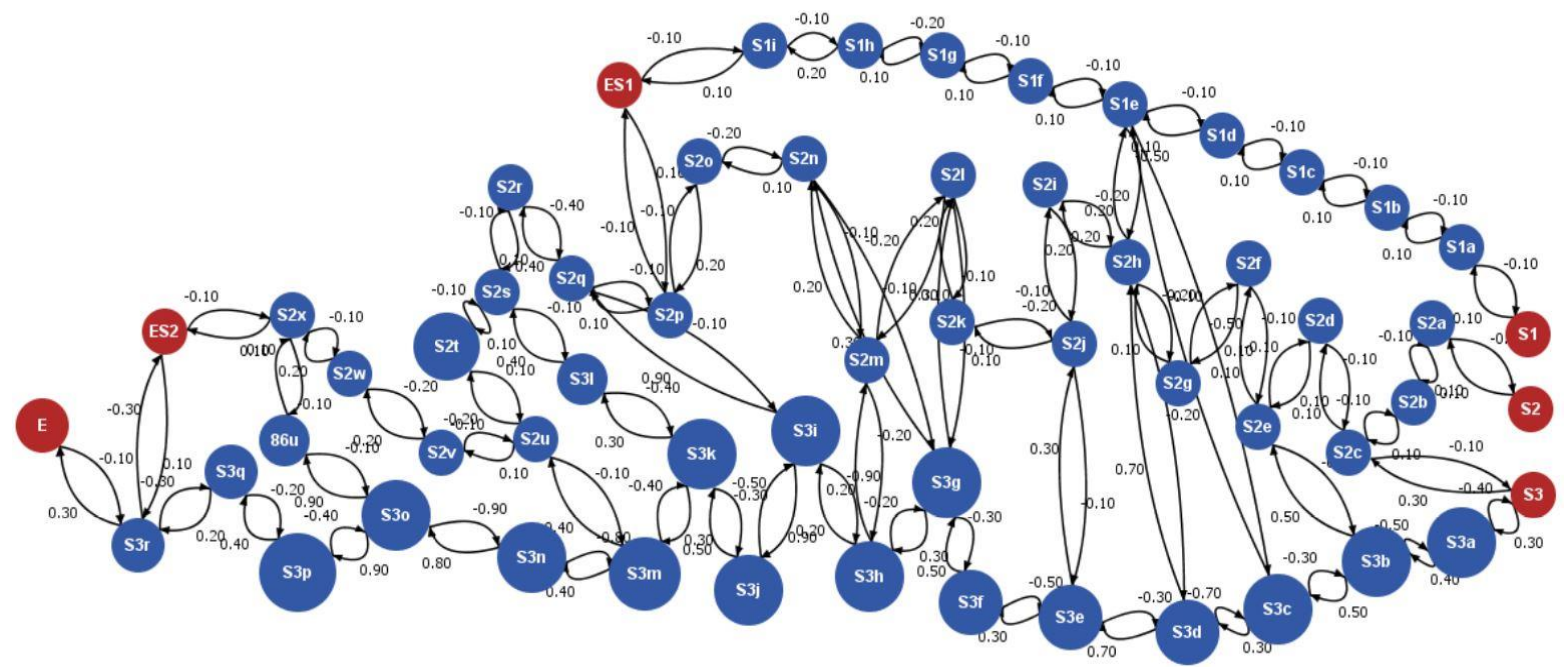

Fig. 7 FCM model of road traffic flow

In Fig. 7, an illustration of the FCM with initialized concepts and weights is shown. In terms of the initial state of the concepts, FCM begins to simulate the performance of the process. In every running step of the FCM, the state of concepts is computed according to step 9 (i.e., in the model simulation steps, see section 3 ). These steps are considered as those of a process in which the values of the defined concepts are analyzed. The value of every concept is assigned by considering all involved causal connection weights directed towards the concept and multiplying every weight by the value of the concept, which causes the connection, then adding the last value of every concept. In this simulation, a sigmoid function with $\lambda>0$ was employed; therefore, the outcomes assumed values in the interval $[0,1]$.

The FCM for road traffic flow modeling with initial vector values $A_{0}$ simulates the state of the system, and the values of the concepts for the desired iteration are illustrated in Fig. 8. The configuration of the FCM reasoning process was set on the 
Developing a macroscopic model based on fuzzy

cognitive map for road traffic flow simulation

stopping criterion when the fixed-point attractor was reached with 0.001 accuracy, and the values of concepts converged to the equilibrium region after seven iterations.

Table 2 presents the values of the concepts for these iterations. The results of the proposed FCM model of the road traffic flow follows a straightforward rule where each freeway segment is represented by a concept, and the density of each segment is signified by the computed values. As it can be observed, the values of these concepts mostly do not alter after the sixth step. Once the FCM reaches the equilibrium state, the new values of concepts are exchanged by the equivalent real values and vice versa. Evaluating the simulation results in the main segments of link $S_{3}$ as the most demanded part of the selected network illustrates the values of $S_{3}, S_{3 a}, S_{3 c}, S_{3 d}, S_{3 e}, S_{3 i}, S_{3 j}, S_{3 h}, S_{3 k}$, and $S_{3 n}$, which formed the only group among all segments where after reaching a peak a downward trend followed. At the same time, the other five segments, namely, $S_{3 b}, S_{3 f}, S_{3 g}, S_{3 l}$, and $S_{3 m}$ showed constant increasing behavior. In this group of segments, when the FCM reaches the equilibrium point, the simulated density values of segments are transmitted to the real system and set the corresponding connected nodes. Finally, the FCM receives the simulated measurements from segments interactions, it interacts, then reaches an equilibrium point and transmits the density values of concepts to the whole model, and this iterative process continues.

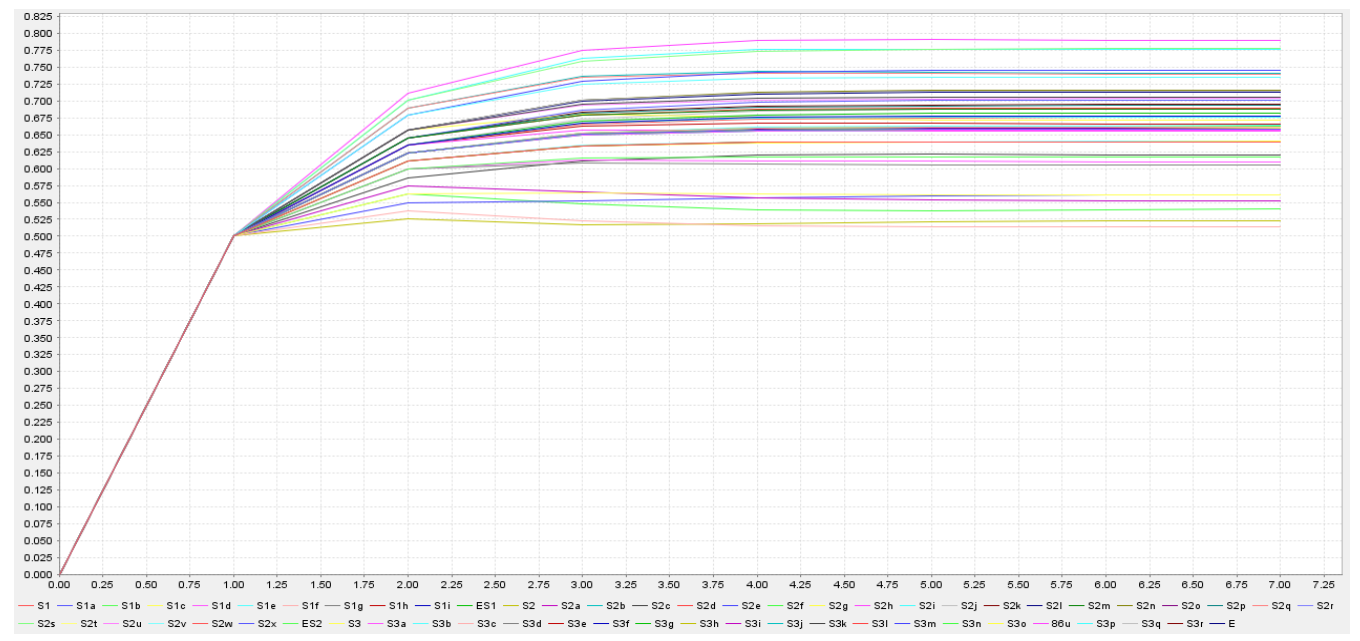

Fig. 8 Concepts' values variation in the FCM inference process

TABLE 2: PARTIAL CONCEPTS' VALUES OF LINK $\mathrm{S}_{3}$ IN THE FIRST SIMULATION STEPS

\begin{tabular}{|c|c|c|c|c|c|c|c|c|c|c|c|c|c|c|c|}
\hline Step & S3 & S3a & S3b & S3c & S3d & S3e & S3f & S3g & S3h & S3i & S3j & S3k & S3I & S3m & S3n \\
\hline 0 & 0 & 0 & 0 & 0 & 0 & 0 & 0 & 0 & 0 & 0 & 0 & 0 & 0 & 0 & 0 \\
\hline 1 & 0.5 & 0.5 & 0.5 & 0.5 & 0.5 & 0.5 & 0.5 & 0.5 & 0.5 & 0.5 & 0.5 & 0.5 & 0.5 & 0.5 & 0.5 \\
\hline 2 & 0.5622 & 0.5987 & 0.6225 & 0.5374 & 0.5987 & 0.6341 & 0.6225 & 0.6341 & 0.525 & 0.5744 & 0.69 & 0.5866 & 0.6457 & 0.5498 & 0.5622 \\
\hline 3 & 0.5639 & 0.6121 & 0.6531 & 0.5225 & 0.6089 & 0.6629 & 0.6508 & 0.6702 & 0.5173 & 0.566 & 0.7371 & 0.6114 & 0.6795 & 0.5524 & 0.5476 \\
\hline 4 & 0.5621 & 0.6118 & 0.6611 & 0.5151 & 0.6072 & 0.6668 & 0.6567 & 0.6796 & 0.5183 & 0.5566 & 0.7433 & 0.6195 & 0.6872 & 0.5569 & 0.539 \\
\hline 5 & 0.5614 & 0.6106 & 0.6631 & 0.5135 & 0.6059 & 0.6665 & 0.6577 & 0.6818 & 0.521 & 0.5534 & 0.7424 & 0.6209 & 0.6891 & 0.5601 & 0.5382 \\
\hline 6 & 0.5614 & 0.61 & 0.6635 & 0.5134 & 0.6056 & 0.6661 & 0.6577 & 0.6821 & 0.5225 & 0.553 & 0.7416 & 0.6207 & 0.6895 & 0.5611 & 0.5392 \\
\hline 7 & 0.5614 & 0.6099 & 0.6635 & 0.5134 & 0.6056 & 0.6659 & 0.6577 & 0.6821 & 0.5229 & 0.553 & 0.7413 & 0.6204 & 0.6895 & 0.5611 & 0.5398 \\
\hline
\end{tabular}

An advantage of the proposed FCM road traffic flow model is that it supports performing what-if simulation analysis based on altering the properties of the involved variables, and subsequently, it may be observed how the system behavior might be affected by changes in particular variables. As a most common congestion-related event in the freeways, lane drop is being caused by various possible events such as accidents, maintenance, etc., that can lead to shifting free-flow traffic to a congested state and delayed travel time. Therefore, this scenario was performed in the $S_{3 h}$ segment chosen as one of the highdensity segments in the selected network, with a reproducible recurring congestion event potential forming a bottleneck location. Bottlenecks are parts of segments where traffic congestion is repeatedly evidenced, which possess a reducing capacity on the segment upstream and freely flowing traffic on downstream. They have two main types of dynamics, namely, slow-moving vehicles and frequent accidents, as well as static features, e.g., tunnel entrances [53], [54]. In this scenario, one of the two lanes of $S_{3 h}$ was dropped, and the FCM simulation process started. The results of this simulation can be observed in Table 3. As opposed to the first inference process, where values stop changing after the sixth step, in this case, the values of the concepts mostly continued changing after the sixth step as well.

Although one lane was dropped, the density in the $S_{3 h}$ segment decreased only slightly, which fact shows that density in the remaining lane dramatically increased. In the meanwhile, density value alterations in the corresponding connected nodes $S_{3 g}$ and $S_{3 i}$ with $S_{3 h}$ in link $S_{3}\left(S_{3 g}\right.$ and $S_{3 i}$ can be seen in Fig. 9) are indicated. Their geographical map and the FCM concept representations can be seen in Fig. 6, respectively. The simulation of one lane being dropped shows a downward trend up to $10 \%$ in the density of the subsequent connected segment $\left(S_{3 i}\right)$ compared to the results of the first simulation process in Table 2; whereas, it has a rising impact on the traffic state in the previous segments as the upstream of the sections $S_{3 h}$, where $S_{3 g}$ and $S_{3 f}$ experienced upward trends in their density around $20 \%$ and $5 \%$, respectively. 
TABLE 2: ONE SEGMENT LANE DROPPED IMPACT ON THE NETWORK

\begin{tabular}{ccccccccccccccccc}
\hline Step & S3 & S3a & S3b & S3c & S3d & S3e & S3f & S3g & S3h & S3i & S3j & S3k & S3I & S3m & S3n \\
\hline $\mathbf{0}$ & 0 & 0 & 0 & 0 & 0 & 0 & 0 & 0 & 0 & 0 & 0 & 0 & 0 & 0 & 0 & 0 \\
$\mathbf{1}$ & 0.5 & 0.5 & 0.5 & 0.5 & 0.5 & 0.5 & 0.5 & 0.5 & 0.5 & 0.5 & 0.5 & 0.5 & 0.5 & 0.5 & 0.5 & 0.5 \\
$\mathbf{2}$ & 0.5622 & 0.5987 & 0.6225 & 0.5374 & 0.5987 & 0.6341 & 0.6341 & 0.6341 & 0.5125 & 0.5744 & 0.69 & 0.5866 & 0.6457 & 0.5498 & 0.5622 \\
$\mathbf{3}$ & 0.5639 & 0.6121 & 0.6531 & 0.5225 & 0.6089 & 0.6616 & 0.6676 & 0.6725 & 0.4983 & 0.5654 & 0.7371 & 0.6114 & 0.6795 & 0.5524 & 0.5476 \\
$\mathbf{4}$ & 0.5621 & 0.6118 & 0.6611 & 0.5151 & 0.6073 & 0.6646 & 0.6752 & 0.6835 & 0.4971 & 0.5555 & 0.7432 & 0.6195 & 0.6872 & 0.5569 & 0.539 \\
$\mathbf{5}$ & 0.5614 & 0.6106 & 0.6631 & 0.5135 & 0.6061 & 0.664 & 0.6865 & 0.7263 & 0.4992 & 0.5321 & 0.7422 & 0.6209 & 0.6891 & 0.5601 & 0.5382 \\
$\mathbf{6}$ & 0.5614 & 0.61 & 0.6635 & 0.5133 & 0.6058 & 0.6634 & 0.691 & 0.8268 & 0.516 & 0.5105 & 0.7413 & 0.6207 & 0.6895 & 0.5611 & 0.5392 \\
$\mathbf{7}$ & 0.5614 & 0.6099 & 0.6635 & 0.5134 & 0.6058 & 0.6632 & 0.691 & 0.8269 & 0.5188 & 0.5015 & 0.7411 & 0.6204 & 0.6895 & 0.5611 & 0.5398 \\
\hline
\end{tabular}

The presented simulations indicated the abilities of the FCM as a practical soft computing method, not only in macroscopic modeling to investigate the overall behavior of road traffic flow but also to capture the interesting and flexible features in terms of examining and monitoring alterations and modifications of the involved parameters that may affect the whole networks of freeways. These characteristics offer valuable information and can contribute to beneficial results related to the traffic engineering field, such as prediction and surveillance of the road traffic flow state in complex and uncertain networks, the estimation of the influence of new road constructions, or the comparison of various alternatives, the prediction of the effects of capacity increase or reduction, and the improvement and assessment of road traffic control associated strategies, detecting prone error locations and optimizing the network itself [5], [22], [35].

\section{CONCLUSION}

The current rapid progress in road traffic flow modeling urges a distinct emphasis on examining the capacities of various soft computing techniques in this field. This research paper proposed a novel macroscopic model of the road traffic flow, based on the FCM approach as one of the emerging soft computing techniques. Alongside being generic, as it can be adopted to most combinations of a macroscopic road traffic flow modeling, this approach introduced a new application of fuzzy cognitive maps in modeling a nonlinear and complex network of freeways for the very first time, with the focus on detecting the reasons of road traffic congestion. Also, sustainability-related objectives can be investigated with this approach as the key argument in designing and managing transportation systems, an approach that affects the potential of improving road traffic control strategies.

It is plausible that all contributions of a macroscopic road traffic flow model cannot be provided by the FCM model, mainly due to the problem complexity, and the obtained results may differ from the real state of the road traffic. However, any estimation technique can inherently include a tradeoff between model performance and operation speed. In this light, FCM provides real advantages; for example, once trained, the road traffic simulation can be performed rapidly and in most cases at an approved level of accuracy. Furthermore, the dataset of the study does not contain all segments that can affect road traffic behavior, but only those where the e-toll network is included. It is worth mentioning that the resolution of the representation of the networks of freeways can be dramatically improved by employing further mapping and data, consequently leading to more accurate - and obviously, more complex - FCM models with refined simulation results.

\section{ACKNOWLEDGMENT}

This research was supported by the National Office of Research, Development, and Innovation grant NKFIH K124055.

\section{REFERENCES}

[1] A. M. Nagy and V. Simon, "Traffic congestion propagation identification method in smart cities," Infocommunications J., vol. 13, no. 1, pp. 45-57, 2021. DoI: 10.36244/ICJ.2021.1.6

[2] L. Alekszejenkó and T. Dobrowiecki, "Adapting it algorithms and protocols to an intelligent urban traffic control," Infocommunications J., vol. 12 , no. 2 , pp. 57-62, 2020. DoI: 10.36244/ICJ.2020.2.8

[3] G. R. Timilsina and H. B. Dulal, "Urban Road Transportation Externalities: Costs and Choice of Policy Instruments," World Bank Res. Obs., vol. 26, no. 1, pp. 162-191, Feb. 2011. DOI: $10.1093 / \mathrm{wbro} / \mathrm{kq} 005$

[4] W. Imran, Z. H. Khan, T. Aaron Gulliver, K. S. Khattak, and H. Nasir, "A macroscopic traffic model for heterogeneous flow," Chinese J. Phys., vol. 63, no. December 2019, pp. 419-435, 2020. DOI: 10.1016/j.aej.2021.06.042

[5] S. K. Zegeye, B. De Schutter, J. Hellendoorn, E. A. Breunesse, and A. Hegyi, "Integrated macroscopic traffic flow, emission, and fuel consumption model for control purposes," Transp. Res. Part C Emerg. Technol., vol. 31, pp. 158-171, 2013. DOI: 10.1016/j.trc.2013.01.002

[6] M. Amini, M. F. Hatwagner, G. C. Mikulai and L. T. Koczy, "An intelligent traffic congestion detection approach based on fuzzy inference system," 2021 IEEE 15th International Symposium on Applied Computational Intelligence and Informatics (SACI), 2021, pp. 97-104, DOI: 10.1109/SACI51354.2021.9465637.

[7] J. G. WARDROP, "ROAD PAPER. SOME THEORETICAL ASPECTS OF ROAD TRAFFIC RESEARCH.," Proc. Inst. Civ. Eng., vol. 1, no. 3, pp. 325-362, 1952. DoI: 10.1680/ipeds.1952.11259

[8] M. Kalinic and J. M. Krisp, "Fuzzy inference approach in traffic congestion detection," Ann. GIS, vol. 25, no. 4, pp. 329-336, 2019. DOI: $10.1080 / 19475683.2019 .1675760$

[9] S. Ardabili, A. Mosavi, and A. R. Várkonyi-Kóczy, "Advances in machine learning modeling reviewing hybrid and ensemble methods," in International Conference on Global Research and Education, pp. 215-227, 2019. DoI: 10.1007/978-3-030-36841-8_21

[10] A. Csikos, Z. J. Viharos, K. B. Kis, T. Tettamanti, and I. Varga, "Traffic speed prediction method for urban networks- an ANN approach," in 2015 International Conference on Models and Technologies for Intelligent Transportation Systems (MT-ITS), vol. 48, pp. 102-108, 2015. DoI: 10.1109/MTITS.2015.7223243

[11] P. Arnesen and O. A. Hjelkrem, "An estimator for traffic breakdown probability based on classification of transitional breakdown events," Transp. Sci., vol. 52, no. 3, pp. 593-602, 2018. DoI: $10.1287 /$ trsc. 2017.0776

[12] W. Zhang et al., "State-of-the-art review of soft computing applications in underground excavations," Geosci. Front., vol. 11, no. 4, pp. 10951106, 2020. DoI: 10.1016/j.gsf.2019.12.003

[13] Y. Yan, L. Wang, T. Wang, X. Wang, Y. Hu, and Q. Duan, "Application of soft computing techniques to multiphase flow measurement: A review," Flow Meas. Instrum., vol. 60, no. November 2017, pp. 30-43, 2018. DOI: 10.1016/j.flowmeasinst.2018.02.017 
[14] L. A. Zadeh, "Soft Computing and Fuzzy Logic," IEEE Softw., vol. 11, no. 6, pp. 48-56, 1994. Dor: 10.1109/52.329401

[15] J. Zhang, F. Wang, K. Wang, W. Lin, X. Xu, and C. Chen, "DataDriven Intelligent Transportation Systems: A Survey," IEEE Trans. Intell. Transp. Syst., vol. 12, no. 4, pp. 1624-1639, Dec. 2011. Dor: $10.1109 /$ TITS.2011.2158001

[16] E. O. Antonio D. Masegosa, Enrique Onieva, Pedro Lopez-Garcia, "Applications of Soft Computing in Intelligent Transportation Systems," in Soft Computing Based Optimization and Decision Models, vol. 360, Springer International Publishing, pp. 153-175, 2018. Dor: 10.1007/978-3-319-64286-4_4

[17] R. Falcone, C. Lima, and E. Martinelli, "Soft computing techniques in structural and earthquake engineering: a literature review," Eng. Struct., vol. 207, no. November 2019, p. 110269, 2020. DoI: $10.1016 / j$.engstruct.2020.110269

[18] S. Nosratabadi, A. Mosavi, R. Keivani, S. Ardabili, and F. Aram, "State of the art survey of deep learning and machine learning models for smart cities and urban sustainability," in International Conference on Global Research and Education, pp. 228-238, 2019. DOI: $10.1007 / 978-3-030-36841-8 \quad 22$

[19] M. Lippi, M. Bertini, and P. Frasconi, "Short-term traffic flow forecasting: An experimental comparison of time-series analysis and supervised learning," IEEE Trans. Intell. Transp. Syst., vol. 14, no. 2, pp. 871-882, 2013. Dor: 10.1109/TITS.2013.2247040

[20] H. Hartenstein and L. P. Laberteaux, "A tutorial survey on vehicular ad hoc networks," IEEE Commun. Mag., vol. 46, no. 6, pp. 164-171, 2008. DoI: 10.1109/MCOM.2008.4539481

[21] L. Zhang, Z. Yuan, L. Yang, and Z. Liu, "Recent developments in traffic flow modeling using macroscopic fundamental diagram," Transp. Rev., vol. 40, no. 4, pp. 529-550, 2020. DoI: $10.1080 / 01441647.2020 .1738588$

[22] S. Fulari, A. Thankappan, L. Vanajakshi, and S. Subramanian, "Traffic flow estimation at error prone locations using dynamic traffic flow modeling," Transp. Lett., vol. 11, no. 1, pp. 43-53, 2019. DoI: $10.1080 / 19427867.2016 .1271761$

[23] S. P. Hoogendoorn and P. H. L. Bovy, "State-of-the-art of vehicular traffic flow modelling," Proc. Inst. Mech. Eng. Part I J. Syst. Control Eng., vol. 215, no. 4, pp. 283-303, Jun. 2001. DOI: $10.1177 / 095965180121500402$

[24] K. Nagel, P. Wagner, and R. Woesler, "Still flowing: Approaches to traffic flow and traffic jam modeling," Oper. Res., vol. 51, no. 5, pp. 681-710+837, 2003. Dor: 10.1287/opre.51.5.681.16755

[25] G. E. Cantarella, S. De Luca, M. Di Gangi, R. Di Pace, and S. Memoli, "Macroscopic vs. mesoscopic traffic flow models in signal setting design," 2014 17th IEEE Int. Conf. Intell. Transp. Syst. ITSC 2014, pp. 2221-2226, 2014. DoI: 10.1109/ITSC.2014.6958032

[26] M. H. Lighthill and G. B. Whitham, "II-A Theory of Traffic Flow on Long Crowded Roads," Spec. Rep., no. 79, p. 7, 1964. DoI: $10.1098 /$ rspa.1955.0089

[27] M. Glykas, Fuzzy cognitive maps: Advances in theory, methodologies, tools and applications, vol. 247. Springer, 2010. DoI: 10.1007/978-3-642-03220-2

[28] A. Amirkhani, E. I. Papageorgiou, A. Mohseni, and M. R. Mosavi, "A review of fuzzy cognitive maps in medicine: Taxonomy, methods, and applications," Comput. Methods Programs Biomed., vol. 142, pp. 129-145, 2017. DoI: 10.1016/j.cmpb.2017.02.021

[29] G. Felix, G. Nápoles, R. Falcon, W. Froelich, K. Vanhoof, and R. Bello, "A review on methods and software for fuzzy cognitive maps," Artif. Intell. Rev., vol. 52, no. 3, pp. 1707-1737, 2019. Dor: $10.1007 / \mathrm{s} 10462-017-9575-1$

[30] D. Pradeepkumar and V. Ravi, "Soft computing hybrids for FOREX rate prediction: A comprehensive review," Comput. Oper. Res., vol. 99, pp. 262-284, 2018. Dor: 10.1016/j.cor.2018.05.020

[31] E. Bakhtavar, M. Valipour, S. Yousefi, R. Sadiq, and K. Hewage, "Fuzzy cognitive maps in systems risk analysis: a comprehensive review," Complex Intell. Syst., 2020. DoI: 10.1007/s40747-020-00228-2

[32] M. J. Lighthill and G. B. Whitham, "On kinematic waves II. A theory of traffic flow on long crowded roads," Proc. R. Soc. London. Ser. A. Math. Phys. Sci., vol. 229, no. 1178, pp. 317-345, 1955. https://www.jstor.org/stable/99769
[33] C. Pasquale, S. Sacone, S. Siri, and A. Ferrara, "Traffic control for freeway networks with sustainability-related objectives: Review and future challenges," Апnи. Rev. Control, vol. 48, pp. 312-324, 2019. DOI: $10.1016 / \mathrm{j}$.arcontrol.2019.07.002

[34] A. Ferrara, S. Sacone, and S. Siri, "First-order macroscopic traffic models", no. 9783319759593. 2018. DoI: 10.1007/978-3-319-75961-6_3

[35] A. Messmer and M. Papageorgiou, "METANET: a macroscopic simulation program for motorway networks," Traffic Eng. Control, vol. 31, no. 8-9, pp. 466-470, 1990. https://www.researchgate.net/ publication/282285780_METANET_a_macroscopic_simulation program_for_motorway_networks

[36] B. Kosko, "Fuzzy cognitive maps," Int. J. Man. Mach. Stud., vol. 24, no. 1, pp. 65-75, 1986. Dor: $10.1016 / \mathrm{S} 0020-7373(86) 80040-2$

[37] H. J. Payne, "Freflo: a Macroscopic Simulation Model of Freeway Traffic.," Transp. Res. Rec., no. 722, pp. 68-77, 1979.

[38] R. Axelrod, "Structure of Decision: The Cognitive Maps of Political Elites". Princeton: Princeton University Press, 1976 https://www.jstor.org/stable/j.ctt13x0vw3

[39] L. A. Zadeh, "Fuzzy sets," Inf. Control, vol. 8, no. 3, pp. 338-353, 1965. DoI: $10.1016 /$ S0019-9958(65)90241-X

[40] C. D. Stylios and P. P. Groumpos, "Mathematical formulation of fuzzy cognitive maps," Proc. 7th Mediterr. Conf. Control Autom., no. June 1999, pp. 2251-2261, 1999.

[41] G. Nápoles, M. L. Espinosa, I. Grau, and K. Vanhoof, "FCM Expert: Software Tool for Scenario Analysis and Pattern Classification Based on Fuzzy Cognitive Maps," Int. J. Artif. Intell. Tools, vol. 27, no. 7, 2018. DoI: $10.1142 / \mathrm{S} 0218213018600102$

[42] G. Nápoles et al., "Fuzzy Cognitive Modeling: Theoretical and Practical Considerations". Springer Singapore, 2019. Dor: $10.1007 / 978-981-13-8311-3$

[43] B. Kosko, "Hidden patterns in combined and adaptive knowledge networks," Int. J. Approx. Reason., vol. 2, no. 4, pp. 377-393, 1988.

[44] I. Akgun, A. Kandakoglu, and A. F. Ozok, "Fuzzy integrated vulnerability assessment model for critical facilities in combating the terrorism," Expert Syst. Appl., vol. 37, no. 5, pp. 3561-3573, 2010. DoI: 10.1016/j.eswa.2009.10.035

[45] C. D. Stylios and P. P. Groumpos, "Modeling Complex Systems Using Fuzzy Cognitive Maps," IEEE Trans. Syst. Man, Cybern. Part ASystems Humans., vol. 34, no. 1, pp. 155-162, 2004. DoI: $10.1109 /$ TSMCA.2003.818878

[46] E. I. Papageorgiou, "A new methodology for Decisions in Medical Informatics using fuzzy cognitive maps based on fuzzy rule-extraction techniques," Appl. Soft Comput. J., vol. 11, no. 1, pp. 500-513, 2011. Dor: 10.1016/j.asoc.2009.12.010

[47] P. P. Groumpos, "Large Scale Systems and Fuzzy Cognitive Maps: A critical overview of challenges and research opportunities," Annu. Rev. Control, vol. 38, no. 1, pp. 93-102, 2014. Dor: $10.1016 /$ j.arcontrol.2014.03.009

[48] J. L. Salmeron and C. Lopez, "Forecasting Risk Impact on ERP Maintenance with Augmented Fuzzy Cognitive Maps," IEEE Trans. Softw. Eng., vol. 38, no. 2, pp. 439-452, 2012 DOI: $10.1109 /$ TSE.2011.8

[49] F. T. László, T. Péter, "HUNGARY'S ITS NATIONAL REPORT," ITS national report, 2018. [Online]. Available: https://ec.europa.eu/ transport/sites/transport/files/2018_hu_its_progress_report_2017.pdf.

[50] E. C. E. UNECE, "EU transport in figures - Statistical Pocketbook 2020, Number of registered passenger cars in Hungary from 1990 to 2018," European Commission, 2020.

[51] GSMPRO Kft.,"General Terms and Conditions," https://tracker.gsmpro.hu/. 2019.

[52] E. I. Papageorgiou and P. P. Groumpos, "A weight adaptation method for fuzzy cognitive map learning," Soft Comput., vol. 9, no. 11, pp. 846-857, 2005. DoI: 10.1007/s00500-004-0426-z

[53] R. L. Bertini and M. T. Leal, "Empirical study of traffic features at a freeway lane drop," J. Transp. Eng., vol. 131, no. 6, pp. 397-407, 2005. Dor: 10.1061/(ASCE)0733-947X(2005)131:6(397)

[54] S. M. S. Seliman, A. W. Sadek, and Q. He, "Automated Vehicle Control at Freeway Lane-drops: a Deep Reinforcement Learning Approach," J. Big Data Anal. Transp., vol. 2, no. 2, pp. 147-166, 2020. Dor: $10.1007 / \mathrm{s} 42421-020-00021-0$ 
Developing a macroscopic model based on fuzzy cognitive map for road traffic flow simulation
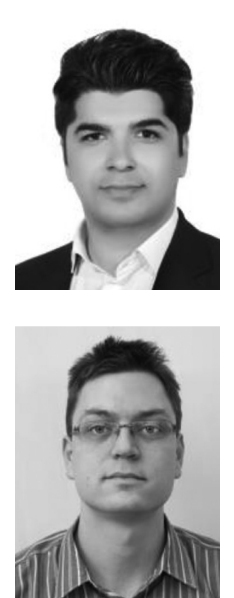

Mehran Amini is a Ph.D. candidate in computer science in the Department of Information Technology at Széchenyi István University, Győr, Hungary. He has almost a decade of professional data analysis expertise, primarily in business intelligence. Computational intelligence and machine learning algorithms in modeling complex systems and risk analysis are among his main research interests. He also teaches Bioinformatics and IT project management.

Miklos F. Hatwagner is an Associate Professor in the Department of Information Technology at Széchenyi István University, Győr, Hungary. He holds a Ph.D. in Information Science from Széchenyi István University (September 2013). He has been working for over ten years as a Researcher in several research projects related to the development of novel parallel implementations of various evolutionary algorithms, the effective error handling techniques in distributed environments, and their application. He has been involved in several national research projects. He was also a member of the Hungarian ENUM project team. Later he turned his attention to the Fuzzy Cognitive Maps (FCM), the training and application of them to solve several problems arose in the fields of management, environmental protection, etc. $\mathrm{He}$ is the author or co-author of approx. 50 conference or journal papers. He has over 100 citations from independent researchers (h-index $=8$ in Google Scholar and hindex $=7$ in Scopus). His research interests include evolutionary algorithms, optimization, parallel computing, info-communication, Fuzzy Cognitive Maps, decision support, machine learning.

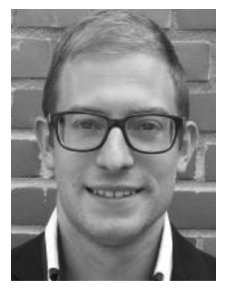

Gergely Cs. Mikulai received the B.Sc. degree in Mechanical Engineering at Budapest University of Technology and Economics (BME) in 2016. He received an M.Sc. degree in Business Development at Óbuda University in 2018. He is currently with Ph.D. Programme of Regional and Economic Sciences with Transdisciplinarity focus. His research interests mainly include route selection issues, using mostly fuzzy signature rule base evaluation.

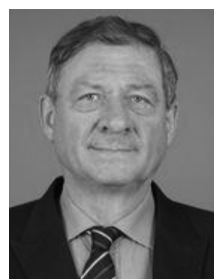

Laszlo T. Koczy received the M.Sc., M.Phil. and Ph.D. degrees from the Technical University of Budapest (BME) in 1975, 1976, and 1977, respectively; and the D.Sc. degree from the Hungarian Academy of Science in 1998. He spent his career at BME until 2001 and from 2002 at Szechenyi Istvan University (Gyor, SZE).

He has been a visiting professor in Australia, Japan, Korea, Austria, Italy, etc. His research interests are fuzzy systems, evolutionary and memetic algorithms, and neural networks, as well as applications in infocommunications, logistics, management, and others. In the last years, he has focused on NP-complete problems, especially route selection and optimization and the application of metaheuristics for approximate solution of such complex tasks. He has published over 775 articles, most of those being refereed papers, and several text books on the subject. His Hirsch-index is 40 by Google Scholar (based on 7300 citations there). 